\section{Getting to grips with chaos}

\section{Michael Berry}

Deterministic Chaos: An Introduction. By Heinz Georg Schuster. Physik-Verlag: 1985. Pp.220. DM98, \$43.75.

IN THE more fully developed sciences, natural laws are expressed as mathematical equations describing how the development of a system is uniquely related to ("determined by") its state at some other, usually earlier, time. (Quantum mechanics is no exception: its celebrated indeterminacy restricts what may be measured in any given state, not the way in which the state evolves.) Determinism does not imply predictability, however, and indeed a wide variety of systems that evolve causally, with no source of external noise, exhibit irregular and even random behaviour. The common property of deterministic chaotic systems is sensitive dependence on initial conditions: as a result of instability in the dynamical equations. two systems initially in very similar states will soon evolve to widely separated ones.

This is not a new idea. Maxwell understood it more than a century ago, and, shortly afterwards, Poincaré began to develop much of the appropriate mathematics. What is new is the emergence of "chaology" as the subject of intensive study in a variety of areas using a variety of techniques. The areas of application range from celestial mechanics (gaps in the asteroid belt) and fluid turbulence to physiological arrhythmia (heart attacks and cot deaths) and the populations in successive generations of an ecosystem. The techniques range from physical experimentation through computer simulation to the purest mathematics.

In his book, Schuster gives a very useful summary of the main ideas of the subject as it now stands. Although a physicist by training and style, he organizes his treatment by the logic of the mathematics, which is based on the concept of a dynamical system. This is any set of equations, describing how a set of variables changes with time, for which a uniqueness theorem guarantees that for each specification of the variables at one time (initial state) there is just one set of values at a later time (final state). This is a very broad framework: "time" may be continuous (as in mechanics, classical or quantum) or

The classic anatomist's textbook The Development of the Vertebrate Skull by Gavin R. deBeer - first published in 1937 - has just been reprinted with a new Foreword written by Brian K. Hall and James Hanken. The book is published by the University of Chicago Press. £18.95, \$22.

clarity and the minimum (but subtle)

discrete (as in the successive generations of an ecosystem); the set of variables may be finite (like the coordinates and velocities of a finite collection of particles) or infinite (like the triplet of velocity components at each position in a fluid).

Most of the book is devoted to simple mappings. where dynamics is reduced to a rule describing how a point in a lowdimensional space (a plane or even a line segment) jumps from "time" $n$ to "time" $n+1$. This is a sensible strategy because much more complicated systems (such as turbulent fluids) appear to have, embedded in them, mappings which organize much of their behaviour. And astonishing complexity can indeed arise from multiple repetition of a simple map. Unravelling this has led to new concepts of wide applicability - strange attractors, Feigenbaum sequences, Lyapunov exponents, Kolmogorov entropy, for example. These and more are explained with maximum

mathematics; abundant illustrations, including beautiful colour plates, also help the reader along.

When there is no friction (a useful idealization in celestial machanics and particle accelerators) different concepts apply. These are well summarized but, as the author admits, his treatment here is less original because many other accounts of this topic are available. Finally, the question of chaotic behaviour in quantum systems is discussed, with the caution appropriate to the unsettled state of the subject.

Students about to begin research into chaos, and practising scientists new to the subject, will find this a book well worth reading.

Michael Berry is Professor of Theoretical Physics in the H.H. Wills Physics Laboratory, University of Bristol. Tyndall Avenue, Bristol BS8 ITZ, UK.

\section{Olfactory work}

\section{Michael Stoddart}

Social Odours in Mammals, Vols 1 and 2. Edited by Richard E. Brown and David W. Macdonald. Clarendon:1985. Pp.882. Vol. I $£ 45, \$ 60 ;$ Vol. $2 £ 32, \$ 45$.

IT Is difficult to know whether this work is a review or a textbook - it has some of the characteristics of both. The editors say they were prompted to gather together contributions from their band of 13 authors because

the growth of theoretically exciting ideas concerning social odours, the scattered publications and expertise [gave us] the feeling that a systematic review of the whole class could provide a realistic perspective and a useful reference work.

Perhaps if they had clarified their objectives rather more they would have decided on what exactly they wanted. In my opinion Social Odours in Mammals is, if anything, a textbook cum reference work, although very different styles are adopted by each author. Its lack of success as a review rests largely on the poor coverage of the literature published during the current decade. Scanning the 20 bibliographies to be found at the end of each chapter one sees very few references post1981 , and most of the 1983 and 1984 references (I found only one of the latter) are to papers written by the chapter authors themselves. Book production is a notoriously slow business, but the price for delay has here been high. Olfactory biology is a rapidly developing field in which an effective review must be up to date.

The textbook quality stems from the books' rigorous taxonomic approach to the essentially functional set of phenomena referred to in the title. It is a great pity there is no subject index (the four indexes included - author, common names, Linnean names and odour sources - occupy almost 50 pages), for there is no way, other than by reading the whole work, to find the latest ideas on, say, scent marking, or species or individual recognition. And no quick way of discovering if advances in specific areas of rodent olfactory biology, to which much space is devoted, are paralleled in other groups. This is an omission which substantially mars the books' usefulness.

What these two volumes do provide is a series of articles examining all that is known about the role of odours in mammals. The rodents are dealt with in four separate chapters - one on the effects of odour on reproductive physiology, one on the Myomorpha, one of the Sciuromorpha and one on the Hystrichimorpha. The primitive eutherians and the primates are given a different treatment; a whole chapter is devoted to a case study of the tree shrew, for example, and a chapter each to the saddle-back tamarin and to human beings. Special treatment for these and not for other well-worked species, such as the golden hamster and the Norway rat, seems inconsistent. Nonetheless, the casehistories provide excellent overviews of the work of two or three leading laboratories in the field. I also particularly enjoyed the chapters on marine mammals and the edentates and pholidotes, as these groups are seldom covered. In all chapters the treatment is thorough, and many tables enhance the presentation.

As an initiate in the field of olfactory biology I find this a useful, if somewhat eclectic, reference work. But I will direct my students to other works to gain a more rounded and up to date impression of the subject.

D. Michael Stoddart is Professor in the Depart ment of Zoology. University of Tasmania. Box 252C. GPO Hobart. Tasmania. Australia 7001 . 